\title{
Epitheloid Leiomyosarcoma of the Jejunum Presenting with Intussusception in a Young Adult Male: A Case Report
}

\author{
John O. Obafunwa ${ }^{{ }^{*}}$, Christopher Pritchett ${ }^{2}$, Siva Maikandanathan ${ }^{2}$, Dahiru Adamu Garkuwa ${ }^{2}$ \\ ${ }^{1}$ Department of Pathology and Forensic Medicine, Lagos State University Teaching Hospital, Lagos, Nigeria \\ ${ }^{2}$ Department of Surgery, South Tyneside District Hospital, South Shields, UK \\ Email: joobafunwa@hotmail.com
}

Received December 31, 2012; revised February 1, 2013; accepted February 10, 2013

Copyright (C) 2013 John O. Obafunwa et al. This is an open access article distributed under the Creative Commons Attribution License, which permits unrestricted use, distribution, and reproduction in any medium, provided the original work is properly cited.

\begin{abstract}
A 27-year-old male presented with intestinal obstruction due to a jejuno-jejunal intussusception, 23 years after a childhood nasopharyngeal carcinoma. He was successfully treated for the latter by radiotherapy but subsequently required a mandibuloplasty for presumably facial hypoplasia sequel to the radiotherapy. The present intussusception was resected with wide margins revealing a partly haemorrhagic, polypoid and sessile jejunal tumour measuring $7.7 \times$ $3.5 \times 2.6 \mathrm{~cm}$. Microscopy with extensive immunohistochemical studies revealed a relatively rare and highly malignant epitheloid leiomyosarcoma arising from the muscularis propria with extension to the mucosa and serosa. No metastases were found and post-operative follow-up has so far been uneventful. The present case of a malignant tumour presenting as an intussusception in a young adult, was unrelated to a childhood malignant tumour in the same patient. The former was successfully treated by a wide excision in support of the advocated surgical approach to adult intussusception.
\end{abstract}

Keywords: Epitheloid Leiomyosarcoma; Intussusception; Surgical Resection

\section{Introduction}

Intussusception with consequent intestinal obstruction is not an uncommon encounter in routine surgical practice and tumours of the small bowel are recognised causes. Usually these tumours are benign, and they include gastrointestinal stromal tumours (GIST) [1], adenomatous polyps and adenomyomas [2,3]. Rare non-neoplastic lesions of the small intestine presenting with intussusception include the inflammatory fibrous polyps also referred to as inflammatory pseudotumours [4]. Malignant tumours, particularly those of mesenchymal origin presenting with intussusception, are rarer in the small intestine. These are commonly the gastrointestinal stromal tumours (GISTs) [5-7]. Less common malignant mesenchymal tumours include well differentiated leiomyosarcomas, malignant schwannomas and undifferentiated sarcomas [6,7].

The present authors report a case of epitheloid leiomyosarcoma (leiomyoblastoma) presenting with intussusception in a young adult male. The clinicopathological findings, surgical approach, immunohistochemical studies and prognostic considerations are discussed.

${ }^{*}$ Corresponding author.

\section{Case History}

A 27-year-old male presented with a 3-day history of vomiting and non-radiating mid-abdominal pain. There was past medical history of a malignant neck lesion at the age of four years, which on biopsy was thought to be Hodgkin's lymphoma. Subsequent review suggested a diagnosis of rhabdomyosarcoma, but further expert opinion was said to have confirmed a diagnosis of nasopharyngeal carcinoma. Unfortunately there are no definitive records from the previous hospital and the histopathological slides were not available to the present authors. However, based on the final diagnosis he underwent radiotherapy with good response and he apparently had good developmental growth over the years. He underwent mandibuloplasty for facial hypoplasia supposedly for complications due to the radiotherapy. There was no history of abdominal surgery.

While presenting on this occasion, there was abdominal tenderness without a palpable mass, and his vital signs were normal. Plain abdominal X-ray showed a distended stomach, but the small bowel appeared normal, and he was managed conservatively. On the third day post-admission 
he started vomiting, producing $>1.0 \mathrm{~L}$ of bilious fluid daily. There were no other significant clinical findings and the urea and electrolytes status remained normal. In view of the persistent vomiting a repeat abdominal X-ray was ordered and this showed gastric distension. However a CT-scan of the abdomen showed features of intussusception in the proximal small bowel. He was taken to theatre on the same day and laparotomy revealed a jejuno-jejunal intussusception that was caused by a tumour (Figure 1); a segmental resection with an end-to-end anastomosis was performed. His condition improved post-operatively and he was discharged on the $14^{\text {th }}$ post-operative day.

Laboratory examination of the resected segment of bowel showed a portion of the jejunum measuring 12.0 $\mathrm{cm}$ in length with a sessile polyp in the mid part. The latter measured $7.7 \mathrm{~cm}$ and $3.5 \mathrm{~cm}$ in the longitudinal and transverse axes respectively with a maximum thickness of $2.6 \mathrm{~cm}$ (Figure 2). Cut section shows a haemorrhagic appearance with a cream colour in the basal third (Figure 3). Microscopy revealed a sessile haemorrhagic, poly-

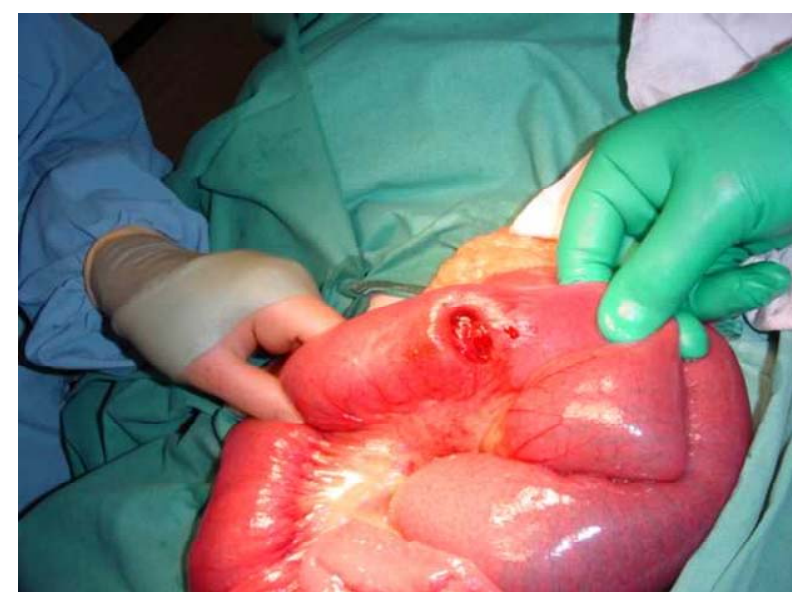

Figure 1. Intra-operative view of the serosal surface of the tumour within the jejunum.

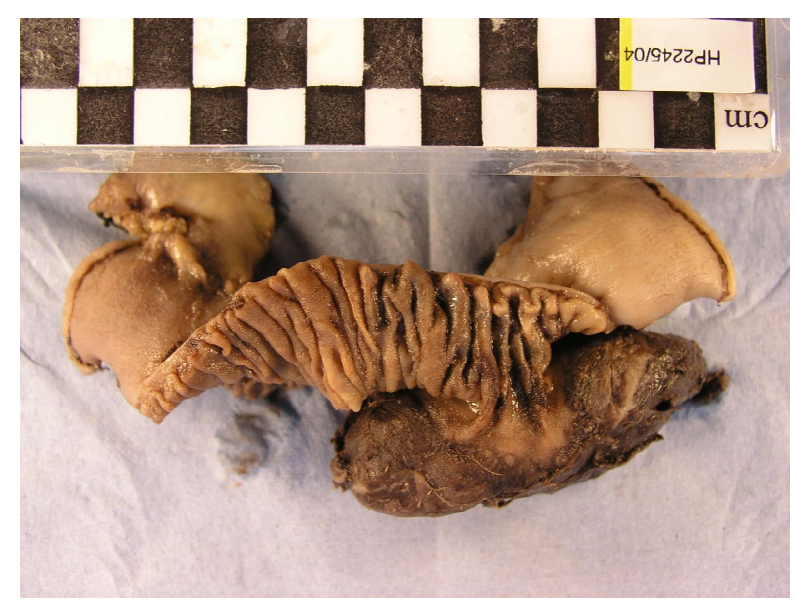

Figure 2. Incised segment of the jejunum showing a sessile polypoid tumour. poid and highly vascularised tumour consisting of sheets of essentially non-cohesive small round fairly uniform cells with indistinct cytoplasm. Their nuclei are minimal pleomorphic, often vesicular and with large prominent nucleoli. Up to 40 mitotic figures are seen per 10 high power fields. The central part of the polyp shows the tumour to have eroded through to the mucosal surface with extensive haemorrhagic infarction (Figure 4(a)). The tumour appears to originate from the muscularis propria but has involved almost the entire bowel wall with virtual extension to the serosal surface in parts (Figure 4(b)). A moderate infiltrate of lymphocytes and a number of plasma cells are seen. Occasional tumor cells showed a spindly appearance.

Immunohistochemical studies revealed reactivity for smooth muscle actin (SMA) (Figure 5) and Vimentin (Figure 6). There was no reactivity for epithelial membrane antigen (EMA), CD34, cytokeratins AE1/AE3, CK7 and CK20. The tumour was also negative for Melan A, Ber EP4, S-100, Factor VIII, Desmin and CD117 (ckit). Leucocyte common antigen (LCA) and T-cell markers CD3, and B-cell markers CD20 and CD39a showed positivity among lymphocytes in the background. Immunohistochemistry for EBV and in-situ hybridization for EBV showed only very occasional positive cells. Utilising Ki67, the tumour showed a proliferation rate of approximately $80 \%$. Based on the above immunoprofile a diagnosis of epitheloid leiomyosarcoma was made, to the exclusion of a malignant GIST, anaplastic lymphoma and malignant melanoma.

\section{Discussion}

Abdominal CT-scan was of immense value in making the clinical diagnosis of intussusception in the present case. This imaging technique remains the most useful tool in

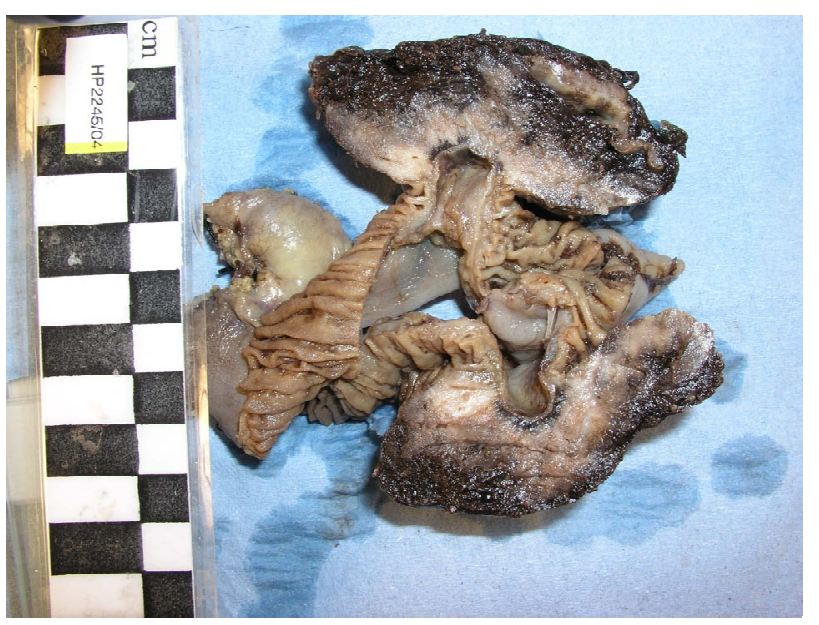

Figure 3. Cut surface of the tumour post-fixation, showing a haemorrhagic upper two-thirds and a cream lower third. Note tumour extension into the subserosa. 


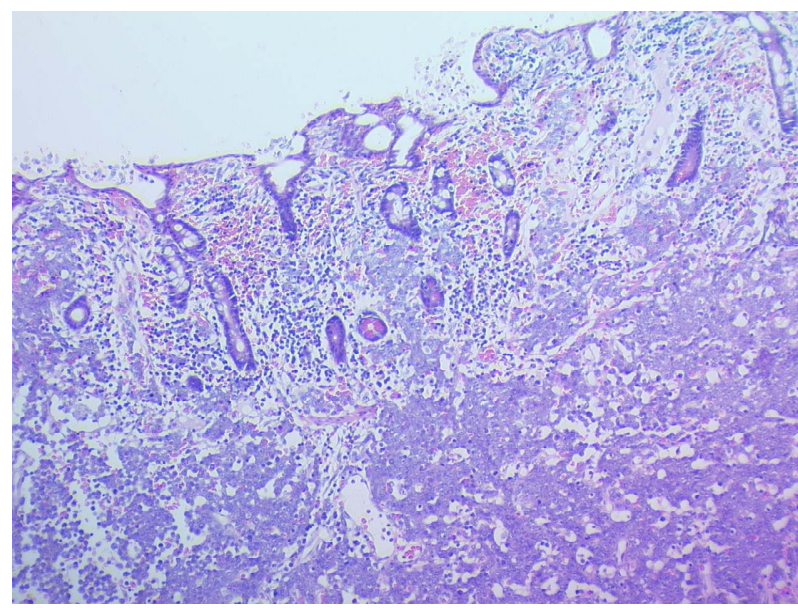

(a)

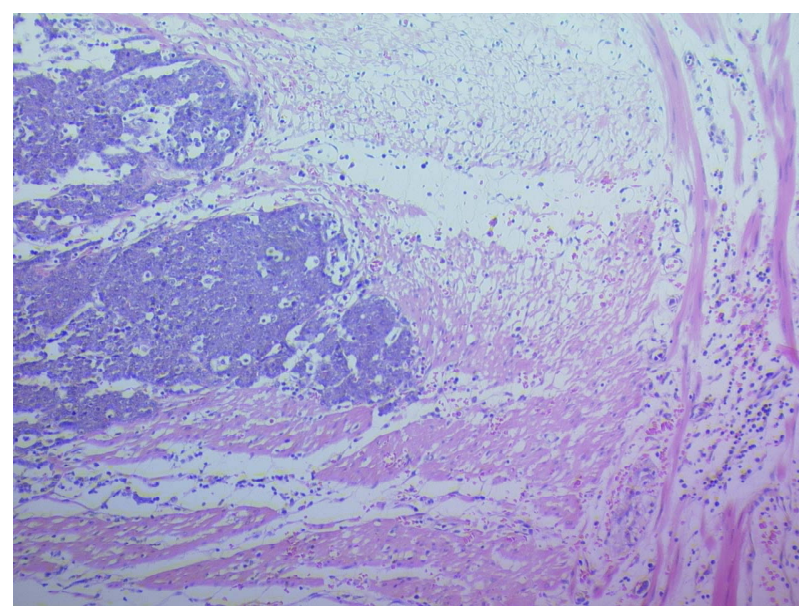

(b)

Figure 4. (a) Sheets of epitheloid neoplastic cells within the mucosa. Note the erosion onto the mucosal surface on the upper left side (Haematoxylin \& Eosin stain $\times 250$ ); (b) Sheets of epitheloid neoplastic cells infiltrating the muscularis propria. The serosal surface is towards the right side (Haematoxylin \& Eosin stain $\times 250$ ).

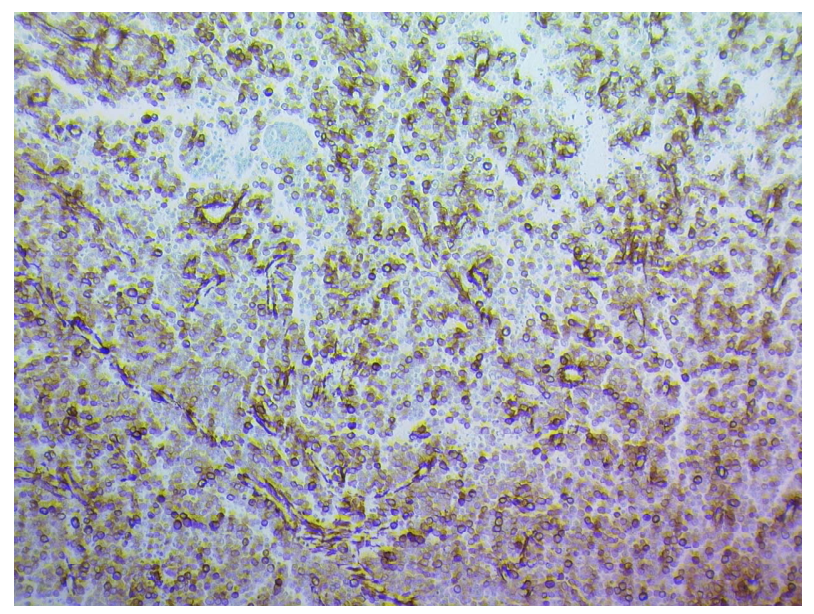

Figure 5. Immunohistochemical stain showing tumour cells exhibiting strong reactivity for smooth muscle actin (SMA).

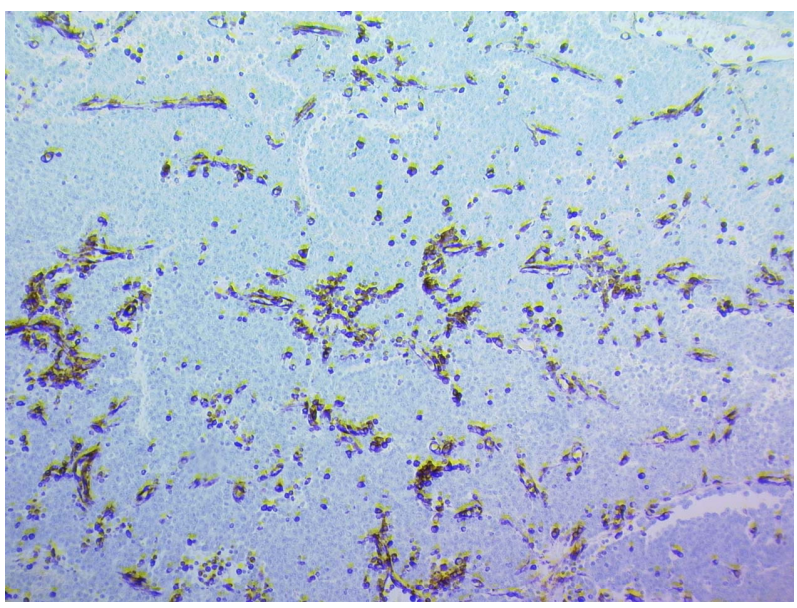

Figure 6. Immunohistochemical stain showing tumour cells exhibiting reactivity for vimentin.

making this diagnosis $[8,9]$ apart from being capable of evaluating tumour location, size, permeation of adjoining organs and locating metastases.

Histopathological examination of the resected tumour revealed a highly malignant tumour with an immunoprofile compatible with an epitheloid leiomyosarcoma. The surgical decision to resect conforms with the recommendation that except for pre-operatively histologically proven benign tumors, intussusception in adults should not be reduced; they should be resected $[8,10]$. This management approach contrasts that in the paediatric age group where a diagnosis of a malignant tumour is likely to be encountered. The only other exception to the adult approach is when there is the likelihood that a short gut syndrome could result from the bowel resection. Generally the approach at surgery would have to be individualised, taking into consideration the findings at laparotomy, such as infarcted bowel segments.

Epitheloid smooth muscle tumours show a predilection for the abdomen. In a review of 155 cases of leiomyoblastoma by Pizzimbono et al. (1973), 145 were found in the stomach, four in the omentum and mesentery, three in the small intestine, two in the uterus and one in the retroperitoneum [11]. These tumours often occur in the mid to late adulthood and are rarely seen under the age of 20 years; it is commoner in males [12,13]. The present case is one of the rare instances where the tumour presents in the jejunum, and extensive immunohistochemical studies were utilised to establish the diagnosis. This further supports the view that a resection should be surgical approach in cases of intussusception in adults.

The tumour cells generally contain a combination of fusiform and epitheloid cells; epitheloid changes could be focal or widespread. The present case showed only a very small focus of spindly cells. The terminology epitheloid leiomyosarcoma was used to encompass malignant smooth muscle tumours consisting of sheets and 
clusters of epitheloid cells. These tumours display pleomorphism and mitotic activities. The presence of up to 40 mitotic figures per 10 high power fields in the present case indicates a highly malignant tumour and conesquently a greater chance of metastases $[14,15]$.

Epitheloid leiomyosarcomas could display some variations in immunohistochemical patterns due to whether frozen or fixed samples are used, the type of tissue fixation methods, and which of the various antibodies are employed [15-18]. It is generally agreed that epitheloid smooth muscle tumours do not express muscle antigens like normal smooth muscle, and neural markers are generally negative [19]. The tumour is generally negative for antibodies directed at non-myogenic phenotypes such as the epithelial membrane antigen (EMA), cytokeratins (AE1/AE3, CK7, CK20), carcinoembryonic antigen (CEA), S-100 and factor VIII $[20,21]$. However, they all express vimentin, a feature that was noted in the present case. All the eight cases studied by Cerilli and Wick (2002) were vimentin positive [22]. They also showed the presence of either muscle specific actin or desmin; only in two cases did the tumour express both phenotypes. This observation is in contrast to the pattern among the spindly type of leiomyosarcoma. Suster (1994) made a similar observation [20]. These findings support ultrastructural observations of reduced or poorly organized myofilaments structures in most lesions [22].

While the lineage of this tumour could still be described as complicated due to few variations in the immunohistochemical staining patterns, they definitely differ significantly from the usual leiomyosarcomas found in the uterus and soft tissues. However, the presence of actins suggests smooth muscle differentiation. It is important to also distinguish this tumour from malignant GISTs, which exhibit various forms of differentiations. These include those arising from smooth muscle, neural, mixed smooth muscle and neural, as well as those showing neither forms of differentiation [23]. Generally, these tumours show strong diffuse staining for vimentin, CD34 (30\% - 70\%), SMA (20\% - 25\%), desmin $(10 \%-33 \%)$, S-100 (5\%) and keratins (5\%). However, an emerging diagnostic marker for GISTs is the expression of a c-kit proto-oncogene present in the interstitial cells of Cajal (CD117); this is absent in schwannomas, kaposi's sarcoma and solitary fibrous tumours $[24,25]$. The present case was negative for CD117. Malignant GISTs show up to $85 \%$ positivity for CD117 in most studies [23]. It is also noteworthy that malignant myogenic tumours are usually negative for CD34 in contrast to GISTs [16]. Furthermore, while ganglion cells and schwannomas are CD34 negative, they stain with S - 100 in the majority of cases [26]. The application of these immunostains in the present case, helped to exclude various differential diagnoses. The immunoprofile showed no semblance to ma- lignant lymphoma, rhabdomyosarcoma or nasopharyngeal carcinoma. This is important in view of the reported past medical history.

As earlier indicated the tumour in the present case is highly malignant with a potential for metastases. However none was seen on CT-scan or during surgery. Metastases are usually to the liver and peritoneal surfaces, and the 10-year survival rates are significantly better for tumours arising in the stomach (74\%) compared to those in the intestine $(17 \%)$. It is noteworthy that the surgical sample showed a tumour that had virtually extended to the peritoneal surface, which undoubtedly requires that this patient be closely monitored.

\section{Conclusion}

The authors describe a rare case of highly malignant jejunal epitheloid leiomyosarcoma in a young adult male who presented with a jejuno-jejunal intussusception. The approach at surgery was as recommended for adults and the patient recovered well post-operatively. The histological and immunohistochemical features are consistent with a primary tumour that is unrelated to previous childhood tumour that was treated over twenty years ago. The new tumour is rare in the small intestine and diagnosis depends on extensive immunohistochemical studies. The diagnosis further justifies the need to resect segments of the bowel in cases of adult intussusception. While there were no obvious metastases, the prognostic features are such that a close follow-up is mandatory in order to ensure early detection of possible metastases.

\section{REFERENCES}

[1] N. Hoshino, T. Murata, K. Oka, K. Hoshino, S. Sekoguchi, T. Suga, A. Nishimura, M. Kato and M. Hamada, "Gastrointestinal Stromal Tumours of the Small Intestine That Expressed c-Kit Protein,” Internal Medicine, Vol. 39, No. 11, 2000, pp. 914-919. doi:10.2169/internalmedicine.39.914

[2] J. S. Lee, H. S. Kim, J. J. Jung and Y. B. Kim, “Adenomyoma of the Small Intestine in an Adult: A Rare Cause of Intussusception,” Journal of Gastroenterology, Vol. 37, No. 7, 2002, pp. 556-559. doi:10.1007/s005350200086

[3] H. S. Park, S. O. Lee, J. M. Lee, M. J. Kang, D. G. Lee and M. J. Chung, "Adenomyoma of the Small Intestine: Report of Two Cases and Review of the Literature,” $\mathrm{Pa}$ thology International, Vol. 53, No. 22, 2003, pp. 111-114. doi:10.1046/j.1440-1827.2003.01435.x

[4] J. S. Zager, J. P. Shaw, J. P. Kaufman and G. DeNoto, "Three Cases of Small Bowel Intussusception in Relation to a Rare Lesion: Inflammatory Fibrous Polyps,” Digestive Surgery, Vol. 18, No. 2, 2001, pp. 142-146.

[5] M. Miettinen, M. Sarlomo-Rikala and J. Lasota, "Gastrointestinal Stromal Tumours," Annals of Chirurgica and Gynaecology, Vol. 87, No. 4, 1998, pp. 278-281. 
[6] A. Ouesehal, A. Abdelouafi, B. Belaabidia, F. Essodegui, B. Kadiri and R. Kadiri, "Malignant Stromal Tumours of the Small Intestine: Report of 9 Cases," Journal of Radiology, Vol. 82, No. 1, 2001, pp. 35-40.

[7] M. Miettinen and J. Lasota, "Gastrointestinal Stromal Tumours (GISTs): Definition, Occurrence, Pathology, Differential Diagnosis and Molecular Genetics,” Polish Journal of Pathology, Vol. 54, No. 1, 2003, pp. 3-24.

[8] K. Takeuchi, Y. Tsuzuki, T. Ando, M. Sekihara, T. Hara, T. Kori and H. Kuwano, "The Diagnosis and Treatment of Adult Intussusception,” Journal of Clinical Gastroenterology, Vol. 36, No. 1, 2003, pp. 18-21. doi:10.1097/00004836-200301000-00007

[9] F. Mosca, A. Stracqualursi, A. Persi, O. Zappala, F. Latteri and S. Latteri, "Stromal Tumours of the Small Intestine. Retrospective Analysis of P Cases Surgically Treated,” Chir. Ital, Vol. 54, No. 2, 2002, pp. 133-140.

[10] H. Medina-Franco, A. Ramos-de la Medina and J. AristaNasr, "Intussusception in Adults. Case Report with Uncommon Etiology and Review of Literature," Review of Gastroenterology Mexico, Vol. 67, No. 2, 2002, pp. 103106.

[11] C. A. Pizzimbono, E. Higa and L. Wise, "Leiomyoblastoma of the Lesser Sac: Case Report and Review of the Literature,” The American Journal of Surgery, Vol. 39, No.12, 1973, p. 692.

[12] A. P. Stout, "Bizarre Smooth Muscle Tumours of the Stomach,” Cancer, Vol. 15, No. 2, 1962, pp. 400-409. doi:10.1002/1097-0142(196203/04)15:2<400::AID-CNC R2820150224>3.0.CO;2-P

[13] F. J. DeCastro, W. R. Olsen and E. R. Littler, "Gastric Leiomyoblastoma in an Adolescent," The American Journal of Surgery, Vol. 123, No. 5, 1972, pp. 614-616. doi:10.1016/0002-9610(72)90235-8

[14] R. W. Byard, J. R. Barr, S. P. Naidoo, et al., "Gastric Stromal Tumours with Epitheloid Features: Clinicopathological Features of 22 Cases," The American Journal of Surgical Pathology, Vol. 3, 1990, p. 281.

[15] T. Ueyama, K.-J. Guo, H. Hashimoto, et al., “A Clinicopathologic and Immunohistochemical Study of Gastrointestinal Stromal Tumours," Cancer, Vol. 69, No. 4, 1992, pp. 947-955. doi:10.1002/1097-0142(19920215)69:4<947::AID-CNCR 2820690419>3.0.CO;2-X

[16] M. Miettinen, "Gastrointestinal Stromal Tumours: An Immunohistochemical Study of Cellular Differentiation," American Journal of Clinical Pathology, Vol. 89, No. 5,
1988, pp. 601-610.

[17] Y. Tsutsumi and H. Kubo, "Immunohistochemistry of Desmin and Vimentin in Smooth Muscle Tumours of the Digestive Tract,” Acta Pathologica Japonica, Vol. 38, No. 4, 1988, pp. 455-469.

[18] P. L. Newman, C. Wadden and C. D. M. Fletcher, “Gastrointestinal Stromal Tumours: Correlation of Immunophenotype with Clinicopathologic Features,” The Journal of Pathology, Vol. 164, No. 2, 1991, pp. 107-117. doi:10.1002/path.1711640204

[19] A. M. Pike, R. V. Lloyd and H. D. Appelman, "Cell Markers in Gastrointestinal Stromal Tumours," Human Pathology, Vol. 19, No. 7, 1988, pp. 830-834. doi:10.1016/S0046-8177(88)80267-3

[20] S. Suster, "Epitheloid Leiomyosarcoma of the Skin and Subcutaneous Tissue: Clinicopathologic, Immunohistochemical, and Ultrastructural Study of Five Cases," The American Journal of Surgical Pathology, Vol. 18, No. 3, 1994, pp. 232-240.

doi:10.1097/00000478-199403000-00002

[21] F. Lopez-Barea, J. L. Rodriguez-Peralto, S. Sanchez-Herrera, et al., "Primary Epitheloid Leiomyosarcoma of Bone: Case Report and Literature Review," Virchows Archiv, Vol. 434, No. 4, 1999, pp. 367-371.

[22] L. A. Cerilli and M. R. Wick, "Immunohistology of Soft Tissue and Osseous Neoplasia,” In: D. J. Dabbs, Ed., Diagnostic Immunohistochemistry, Churchill Livingstone, New York, 2002, pp. 59-112.

[23] N. S. Goldstein and J. F. Silverman, “Immunohistochemistry of the Gastrointestinal Tract, Pancreas, Bile Ducts, Gallbladder and Liver,” In: D. J. Dabbs, Ed., Diagnostic Immunohistochemistry, Churchill Livingstone, New York, 2002, pp. 333-406.

[24] M. Sarlomo-Rikala, A. J. Kovatich, B. S. Barusevicius and M. Miettinen, "CD117: A Sensitive Marker for Gastrointestinal Stromal Tumours That Is More Specific than CD34,” Modern Pathology, Vol. 11, No. 8, 1998, pp. 728734.

[25] K. Sicar, B. R. Hewlett, J. D. Huizinga, et al., "Interstitial Cells of Cajal as Precursors of Gastrointestinal Stromal Tumours," The American Journal of Surgical Pathology, Vol. 23, No. 4, 1999, pp. 377-389.

[26] M. Miettinen, M. Virolainen and S. R. Maarit, "Gastrointestinal Stromal Tumours-Value of CD34 Antigen in Their Identifycation and Separation from True Leiomyomas and Schwannomas," The American Journal of Surgical Pathology, Vol. 19, No. 2, 1995, pp. 207-216. 